\title{
Dietary intake of Brazilian adolescents
}

\author{
Catarina Machado Azeredo ${ }^{1, *}$, Leandro Fornias Machado de Rezende ${ }^{2}$, \\ Daniela Silva Canella ${ }^{3}$, Rafael Moreira Claro ${ }^{4}$, Inês Rugani Ribeiro de Castro ${ }^{5}$, \\ Olinda do Carmo Luiz ${ }^{2}$ and Renata Bertazzi Levy ${ }^{2}$ \\ ${ }^{1}$ Federal University of Uberlandia, School of Medicine (Universidade Federal de Uberlândia, Faculdade de \\ Medicina), Av. Pará $n^{\circ}$ 1720, Bloco 2U, Sala 20, Campus Umuarama, Bairro Umuarama, Uberlândia, MG, Brazil \\ 38.405-320: ' ${ }^{2}$ niversity of Sao Paulo, School of Medicine, São Paulo, SP, Brazil: ${ }^{3}$ University of Sao Paulo, School \\ of Public Health, São Paulo, SP, Brazil: ${ }^{4}$ Federal University of Minas Gerais, Nursing School, Belo Horizonte, \\ $M G$, Brazil: ${ }^{5}$ Rio de Janeiro State University, Nutrition Institute, Rio de Janeiro, RJ, Brazil
}

Submitted 31 December 2013: Final revision received 16 May 2014: Accepted 17 June 2014: First published online 4 August 2014

\begin{abstract}
Objective: To analyse the dietary intake of Brazilian adolescents and investigate its association with sociodemographic factors as well as health-risk and healthprotective behaviours.

Design: Cross-sectional study.

Setting: The study was based on data supplied by the National Survey of Schoolchildren's Health (2012) on sociodemographic factors, dietary intake and health-risk and health-protective behaviours of schoolchildren in Brazil. A nutritional scale was elaborated combining markers of healthy and unhealthy diets. Poisson regression analysis was applied to investigate the association between the sociodemographic factors and regular intake ( $\geq 5$ times/week) of selected foods; linear regression analysis was applied to investigate the association of sociodemographic and behavioural factors with nutritional scale score.

Subjects: A total of 109104 adolescents attending the ninth year of education at 2842 schools in Brazil.

Results: Fewer than $30 \%$ of the adolescents consumed raw or cooked vegetables on a regular basis, whereas more than one-third reported regular intake of sweets, soft drinks and sweet biscuits. Adolescents from the southern area and the oldest ones were those most exposed to inadequate dietary intake. The nutritional scale average score was higher in the students attending public school and exhibited a positive correlation with protective behaviours, such as being physically active, having meals with parents and eating breakfast, and a negative correlation with risk behaviours such as eating while studying or watching television and having smoked, drunk alcohol or used other drugs in the previous $30 \mathrm{~d}$.

Conclusions: The results indicate an association between undesirable nutritional habits and other risk behaviours among Brazilian adolescents.
\end{abstract}

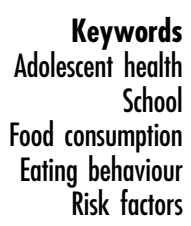

Although chronic non-communicable diseases (NCD) are a matter of great public health concern worldwide, they affect developing countries more significantly ${ }^{(1)}$. In Brazil, NCD cause approximately $70 \%$ of deaths and the prevalence of their main risk factors has shown an unfavourable tendency. These facts make it necessary to implement surveillance programmes to form the basis of timely and strategic interventions ${ }^{(2)}$.

Inadequate dietary habits stand out among the risk factors common to NCD as a whole. Surveys on the types of food available in Brazilian households conducted in the past three decades have shown a steady increase of ultraprocessed foods and a significant reduction of natural or minimally processed foods and cooking ingredients in all socio-economic classes and geographical regions ${ }^{(3,4)}$. That tendency is a serious cause for concern because some features intrinsic to ultra-processed foods (high energy density, free sugar, sodium, total fat and saturated fat; low protein and fibre) are associated with excessive weight gain and increased risk for $\mathrm{NCD}^{(5-7)}$.

Although changes in dietary habits are required in all age ranges, adolescence should be a focus of particular attention because the changes in lifestyle and the development of dietary habits in that stage of life have striking effects $^{(8)}$. Owing to the high prevalence of risk behaviours among adolescents ${ }^{(9)}$ and the future benefits afforded by 
the development of healthy habits at that life stage ${ }^{(10)}$, monitoring the health risk factors in that population is key for the promotion of public health.

In that regard, the first National Survey of Schoolchildren's Health (Pesquisa Nacional de Saúde do Escolar (PeNSE)) was conducted in 2009 by the Health Ministry in partnership with the Brazilian Institute of Geography and Statistics. That survey assessed schoolchildren residing in the capitals of the twenty-six Brazilian states and Federal District and attending the last year of middle school (grade 9). The second PeNSE, which was conducted in 2012, took several health-risk and health-protective factors into consideration and extended the targeted population to include adolescents representative not only of the state capitals, but also of the whole country and in each of its five major geographical areas ${ }^{(11)}$.

The results of the first PeNSE showed that, as a whole, the regular intake of vegetables and fruits by adolescents residing in state capitals was low, while the frequency of foods considered to be markers of an unhealthy diet was high, with some differences according to sociodemographic characteristics $^{(12)}$. By analysing PeNSE 2012 it is possible to identify whether the pattern described for the students from state capitals occurs in the country as a whole and whether there are differences among the five geographic regions and among state capitals and other cities. Therefore, the aim of the present study was to analyse the nationwide and regional food intake patterns of adolescents based on PeNSE 2012 data and to investigate their association with sociodemographic features and some types of behaviours (such as physical activity and use of tobacco, alcohol and other drugs).

\section{Methods}

\section{Study population, sampling and data collection}

The present study was based on data supplied by PeNSE $2012^{(11)}$. The study population comprised adolescents attending the last year of private and public middle schools (grade 9) in Brazil.

The design of PeNSE 2012 ensures sample representativeness relative to the country as a whole, the country's five major geographical areas and the twenty-six state capitals and Federal District. The sample was selected from the 2010 School Census database using a complex design that included stratification per cluster and multistage selection. The sampling strata corresponded to each of the twenty-six state capitals and Federal District, in addition to the five major geographical areas. The procedure for selection of the sampling units applied to the state capitals was different from the procedure used for the set composed of the remainder of counties in each area. In the former case, the primary sampling units were schools and the secondary sampling units were school classes. In the remainder of the counties, primary sampling units were county clusters (established according to their homogeneity and geographical proximity) and secondary sampling units were schools, while the school classes composed tertiary sampling units. The procedure for school and class selection was similar in both cases. The odds for school selection were proportional to the school size (total number of ninth-year classes), while the classes in each school were chosen by simple random selection. Two classes were selected from the schools with three or more ninth-year classes, and one class was selected from the schools with one or two ninth-year classes. All of the students enrolled in the selected classes were invited to participate in the study ${ }^{(11)}$.

From the total number of schools selected to compose the sample ( $n$ 3004), 162 were not considered for analysis owing to several reasons, including lack of ninth-year classes, strikes at the time of data collection and the school board's refusal to participate. On the days of data collection, $84 \%$ (110873) of the total number of students attended school, but 1651 refused participation and 118 did not report their gender or age and thus were excluded from analysis (total response rate of approximately $83 \%$ ). The sample was reweighted to represent the students enrolled in the ninth year of middle school who attended school on a regular basis. Therefore, the present article describes the data corresponding to 109104 students from 2842 schools. Further details on the sampling process are available in the PeNSE report ${ }^{(11)}$.

PeNSE 2012 used a self-reported structured questionnaire available as a smart phone application, which included the following thematic modules: sociodemographic characteristics, occupation, diet, body image, physical activity, smoking, use of alcohol and other drugs, support network (family and friends), hygienic habits, mental health, oral health, asthma, sexual behaviour, violence and accidents, and use of health-care services.

\section{Description of variables and construction of indicators}

The dietary intake was assessed using a validated questionnaire $^{(13)}$ based on the frequency of intake in the previous $7 \mathrm{~d}$ of each of the following eleven groups of foods: (i) beans; (ii) raw vegetables; (iii) cooked vegetables; (iv) natural fruits; (v) milk; (vi) soft drinks (soda); (vii) sweets; (viii) sweet biscuits; (ix) bagged salty snacks; (x) fried salty snacks; and (xi) processed meats. The structure of the questions was: 'During the last seven days, how often (on how many days) have you eaten (food)', and options of answer were: 'I haven't eaten (food) in the last seven days', 'one day, in the last seven days', 'two days, in the last seven days', 'three days, in the last seven days', 'four days, in the last seven days', 'five days, in the last seven days', 'six days, in the last seven days' and 'every day in the last seven days'. The first five items were considered markers of healthy diet and the remainder of the items markers of unhealthy diet ${ }^{(5,14)}$. 
For the purpose of data analysis, food intake was categorized by whether a food type was regularly consumed (at least five times in the previous $7 \mathrm{~d}$ ) ${ }^{(15)}$ and by means of a nutritional score based on the frequency of intake of each of the eleven food groups. The total score was calculated by adding the partial scores corresponding to the weekly frequency of intake of the food groups as follows: for the healthy diet markers, the scale ranged from 0 (did not consume) to 7 (every day); and for the unhealthy diet markers, the scale ranged from 7 (did not consume) to 0 (every day). Therefore, the total score could vary from 0 to 77 and the higher the score, the better the nutritional quality of the diet. The scale's internal consistency was assessed by item correlation and calculation of Cronbach's coefficient $(\alpha=0 \cdot 7)$. The item correlation value varied from -0.002 to $0 \cdot 40$.

In addition, the frequency of the following behaviours in a regular week was assessed: having lunch or dinner with their parent or guardian, eating breakfast (markers of healthy behaviour); and eating while studying or watching television (marker of unhealthy behaviour). The level of physical activity was estimated based on the time allotted to gym classes at school, sports, leisure physical activities and active commuting in the previous week. The participants who spent at least 300 min on those activities were considered active ${ }^{(16)}$. Other behaviours, such as smoking, drinking alcohol or using drugs at least once in the previous $30 \mathrm{~d}$, were also assessed.

The following sociodemographic variables were considered in the analysis: gender; age range (11-14 and $\geq 15$ years); ethnicity/skin colour (white, black and brown, Asian, American Indian); and mother's educational level (incomplete middle school, complete middle school, complete high school, complete higher education). Finally, we also assessed the type of county of residence (state capital, non-capital), geographical area (North, Northeast, South, Southeast and Mid-west) and school administrative status (public or private).

Multiple imputation by chained equations was used to attribute numerical values to mother's educational level, which had $17 \%$ missing values ( $n$ 18 527). For that purpose, we assumed that the losses were of the missingat-random type, i.e. conditioned to the data corresponding to other variables. The following were considered predictive variables: student's gender; father's educational level; family goods (car, home telephone, mobile phone, number of bathrooms equipped with a shower in the house); and services (housemaid and access to Internet at home). Because multiple imputation is a stochastic procedure, the data were imputed ten times, and the results exhibited satisfactory statistical reproducibility according to Monte Carlo error analysis ${ }^{(17)}$.

\section{Statistical analysis}

First, the prevalence and distribution of the covariables of interest were analysed. Then, the relationships between regular intake ( $\geq 5$ times/week) of each food group and the participants' sociodemographic characteristics, school administrative status, geographical area and county type were assessed by Poisson regression models, whereby adjusted prevalence ratios (PR) with the corresponding $95 \%$ confidence intervals were obtained ${ }^{(18)}$. The relationships between the nutritional scale score and sociodemographic and behavioural variables were assessed by multiple linear regression models. The cut-off point for statistical significance was established as $P=0 \cdot 05$. All analyses were performed using the statistical software package Stata SE version $12 \cdot 1$ and took the complex design of PeNSE 2012 into account.

\section{Ethical aspects}

PeNSE 2012 was approved by the National Commission of Research Ethics (Comissão Nacional de Ética em Pesquisa (CONEP); record no. 16 805); it was performed in accordance with the Declaration of Helsinki and all participants gave their informed consent. Its database was made publicly available at the Brazilian Institute of Geography and Statistics' website without any information allowing the identification of participants.

\section{Results}

Approximately $80 \%$ of the assessed students attended public schools, and most of them resided in the Southeast area $(44.3 \%)$. The gender distribution was homogeneous, the age range 11-14 years was most common, and most participants were white- or black and brown-skinned. The mothers of about one-third of the participants had a low educational level (incomplete middle school) and onethird had an average educational level (incomplete higher education). Among the healthy diet markers, regular intake of beans (69.9\%) and milk (51.5\%) exhibited the highest proportions, regular intake of cooked vegetables the lowest (13.5\%). Among the unhealthy diet markers, regular intake of sweets $(41.3 \%)$, soft drinks $(33.3 \%)$ and sweet biscuits $(32.5 \%)$ were most prominent. As for foodrelated behaviours, most participants reported regularly ( $\geq 5$ times/week) having meals with their parents, eating while watching television and eating breakfast (Table 1).

Multiple analysis of the indicators of regular intake of healthy foods showed that a smaller proportion of the girls consumed beans $(P R=0.85)$ and milk $(P R=0.90)$ on a regular basis and a slightly larger proportion consumed fruit $(P R=1.04)$ and raw vegetables $(P R=1.09)$ regularly compared with the boys. Older age was associated with a lower proportion of regular intake of beans $(P R=0.96)$ and milk $(\mathrm{PR}=0.94)$ and higher proportion of regular intake of cooked vegetables $(P R=1 \cdot 13)$. Increased mother's educational level was associated with a higher proportion of regular intake of healthy foods, except for beans, which tended to decrease $(P R=0 \cdot 96)$. The proportions of individuals who consumed beans $(P R=1 \cdot 19)$, fruits $(P R=1 \cdot 08)$ 
Table 1 Sociodemographic characteristics, intakes of selected foods and health-risk and health-protective behaviours in Brazilian adolescents attending the ninth year of basic education; PeNSE 2012

\begin{tabular}{|c|c|c|}
\hline \multirow[b]{2}{*}{ Variable } & \multicolumn{2}{|c|}{ Total } \\
\hline & $n$ & $\%$ \\
\hline \multicolumn{3}{|l|}{ Gender } \\
\hline Male & 52015 & $47 \cdot 8$ \\
\hline Female & 57089 & $52 \cdot 2$ \\
\hline \multicolumn{3}{|l|}{ Age range } \\
\hline $11-14$ years & 73343 & 68.4 \\
\hline 15 years or older & 35761 & $31 \cdot 6$ \\
\hline \multicolumn{3}{|l|}{ Ethnicity/skin colour } \\
\hline White & 37674 & $36 \cdot 8$ \\
\hline Black and brown & 62747 & $55 \cdot 6$ \\
\hline Asian & 4821 & 4.1 \\
\hline American Indian & 3790 & 3.5 \\
\hline \multicolumn{3}{|l|}{ Maternal educational level } \\
\hline Incomplete middle school & 37629 & 39.0 \\
\hline Complete middle school & 18978 & $17 \cdot 8$ \\
\hline Complete high school & 35448 & $30 \cdot 7$ \\
\hline Complete higher education & 17015 & 12.5 \\
\hline \multicolumn{3}{|l|}{ Regular intake of foods ( $\geq 5$ times/week) } \\
\hline Beans & 66513 & 69.9 \\
\hline Raw vegetables & 29659 & $26 \cdot 6$ \\
\hline Cooked vegetables & 15075 & 13.5 \\
\hline Fruit & 31645 & $30 \cdot 2$ \\
\hline Milk & 55813 & 51.5 \\
\hline Soft drinks & 35601 & $33 \cdot 3$ \\
\hline Sweets & 44427 & $41 \cdot 3$ \\
\hline Sweet biscuits & 33562 & $32 \cdot 5$ \\
\hline Cold meats & 15239 & 14.6 \\
\hline Fried salty snacks & 16987 & $15 \cdot 8$ \\
\hline Bagged salty snacks & 12662 & $13 \cdot 0$ \\
\hline \multicolumn{3}{|l|}{ Health-risk and health-protective behaviours } \\
\hline Meals with parents ( $\geq 5 \mathrm{~d} /$ week) & 71118 & $66 \cdot 4$ \\
\hline Eating while studying or watching TV ( $\geq 5 \mathrm{~d} /$ week) & 55745 & $53 \cdot 1$ \\
\hline Eating breakfast $(\geq 5 \mathrm{~d} /$ week) & 66579 & 61.9 \\
\hline Smoked in the past $30 \mathrm{~d}$ & 5748 & $5 \cdot 1$ \\
\hline Drank alcohol in the past $30 \mathrm{~d}$ & 27763 & $26 \cdot 1$ \\
\hline Used drugs in the past $30 \mathrm{~d}$ & 2842 & $2 \cdot 4$ \\
\hline Physically active (total activity $\geq 300 \mathrm{~min} /$ week) & 44441 & $39 \cdot 7$ \\
\hline \multicolumn{3}{|l|}{ School administrative status } \\
\hline Public & 86600 & $82 \cdot 8$ \\
\hline Private & 22504 & $17 \cdot 2$ \\
\hline \multicolumn{3}{|l|}{ Geographical area } \\
\hline North & 22774 & 8.0 \\
\hline Northeast & 31301 & $25 \cdot 3$ \\
\hline Southeast & 19660 & $44 \cdot 3$ \\
\hline South & 14878 & $14 \cdot 6$ \\
\hline Mid-west & 20491 & 7.9 \\
\hline \multicolumn{3}{|l|}{ County type } \\
\hline Capital & 61145 & $22 \cdot 4$ \\
\hline Non-capital & 47959 & $77 \cdot 6$ \\
\hline
\end{tabular}

PeNSE, Pesquisa Nacional de Saúde do Escolar (National Survey of Schoolchildren's Health); TV, television.

and cooked vegetables $(\mathrm{PR}=1 \cdot 07)$ were slightly higher among the students attending public schools, while the proportion of individuals who consumed milk $(P R=0.95)$ was lower, compared with students attending private schools. Compared with the North, the Southeast exhibited a greater proportion of students with regular intake of beans and milk, and the Mid-west had a greater proportion of students with regular intake of fruits, raw vegetables and cooked vegetables. The counties that did not contain state capitals had a greater proportion of students who consumed beans $(\mathrm{PR}=1 \cdot 17)$ and a smaller proportion who consumed milk $(P R=0.91)$ on a regular basis compared with the counties that did have state capitals (Table 2).

A higher proportion of girls than boys reported regular intake of all unhealthy diet markers except soft drinks. A higher proportion of older students tended to consume unhealthy foods on a regular basis, except for sweets, compared with their younger counterparts. Small differences were found between ethnicity/skin colour groups: the proportion of regular intake of bagged salty snacks, sweet biscuits and sweets was higher among the blackand brown-skinned and Asian participants compared with the white ones. The increase in maternal education was associated with a higher proportion of regular intake of all foods from this group. The proportions of participants who consumed bagged salty snacks $(\mathrm{PR}=1 \cdot 18)$, sweet biscuits $(\mathrm{PR}=1.08)$ and sweets $(\mathrm{PR}=1.03)$ on a regular basis were higher among the students at public $v$. private schools, while the remainder of the unhealthy diet markers were higher among students from private schools. The Southeast exhibited the highest and the North the lowest proportion of regular intake of unhealthy foods. The counties not containing state capitals exhibited a greater proportion of students who consumed bagged salty snacks $(\mathrm{PR}=1 \cdot 12)$ on a regular basis compared with the counties containing state capitals, while the opposite was found for processed meats $(P R=0.91)$ and soft drinks $(P R=0.92$; Table 3).

The average score on the nutritional scale was $42 \cdot 50$ (sE $0 \cdot 22$ ), and the interquartile range was 36-50. Multiple analysis of the factors associated with the nutritional scale score showed positive associations with a higher frequency of meals eaten with parents, eating breakfast and being active, and negative correlations with female gender, eating while studying or watching television and having smoked, drunk alcohol or used drugs in the previous $30 \mathrm{~d}$. The scores of the participants attending public schools from the Mid-west area were higher than those of the participants attending private schools or residing in the North area (Table 4).

\section{Discussion}

Assessment of a representative sample of students enrolled in the ninth year of Brazilian public and private schools showed that the frequency of regular intake of healthy foods such as fruits and vegetables was low, whereas the regular intake of unhealthy foods such as soft drinks, sweets and sweet biscuits was prevalent. In addition, the positive associations of the nutritional scale score with behaviours protective of health, as well as the negative associations of the score with other risk behaviours for NCD, show that healthy dietary behaviours coexist with other behaviours protecting against NCD in adolescents. This finding shows that there may be a profile of 
Table 2 Proportion of Brazilian adolescents who regularly consumed ( $\geq 5$ times/week) foods considered to be markers of a healthy diet according to sociodemographic variables; PeNSE 2012 Healthy diet markers (consumed $\geq 5 \mathrm{~d} /$ week)

\begin{tabular}{|c|c|c|c|c|c|c|c|c|c|c|c|c|c|c|c|}
\hline \multirow[b]{2}{*}{ Variable } & \multicolumn{3}{|c|}{ Beans } & \multicolumn{3}{|c|}{ Fruit } & \multicolumn{3}{|c|}{ Raw vegetables } & \multicolumn{3}{|c|}{ Cooked vegetables } & \multicolumn{3}{|c|}{ Milk } \\
\hline & $\%$ & $\mathrm{PR}^{*}$ & $95 \% \mathrm{Cl}$ & $\%$ & $\mathrm{PR}^{*}$ & $95 \% \mathrm{Cl}$ & $\%$ & $\mathrm{PR}^{*}$ & $95 \% \mathrm{Cl}$ & $\%$ & $\mathrm{PR}^{*}$ & $95 \% \mathrm{Cl}$ & $\%$ & $\mathrm{PR}^{*}$ & $95 \% \mathrm{Cl}$ \\
\hline \multicolumn{16}{|l|}{ Gender } \\
\hline Male & 74.68 & 1.00 & Ref. & 29.57 & 1.00 & Ref. & 25.59 & 1.00 & Ref. & $13 \cdot 17$ & 1.00 & Ref. & 54.68 & 1.00 & Ref. \\
\hline Female & 65.59 & 0.85 & $0.84,0.86$ & $30 \cdot 79$ & 1.04 & $1.02,1.06$ & 27.47 & 1.09 & $1.07,1.11$ & $13 \cdot 73$ & 1.04 & $1.00,1.07$ & 48.68 & 0.90 & $0.89,0.91$ \\
\hline \multicolumn{16}{|l|}{ Age range } \\
\hline $11-14$ years & 69.93 & 1.00 & Ref. & $30 \cdot 77$ & 1.00 & Ref. & $27 \cdot 20$ & 1.00 & Ref. & $12 \cdot 80$ & 1.00 & Ref. & 53.55 & 1.00 & Ref. \\
\hline 15 years or older & 69.95 & 0.96 & $0.95,0.97$ & 28.98 & 0.98 & $0.96,1.00$ & $25 \cdot 22$ & 0.99 & $0.97,1.01$ & 14.89 & 1.13 & $1.09,1.16$ & $47 \cdot 19$ & 0.94 & $0.92,0.95$ \\
\hline \multicolumn{16}{|l|}{ Ethnicity/skin colour } \\
\hline White & 67.59 & 1.00 & Ref. & $30 \cdot 62$ & 1.00 & Ref. & 28.42 & 1.00 & Ref. & $12 \cdot 71$ & 1.00 & Ref. & $56 \cdot 01$ & 1.00 & Ref. \\
\hline Black and brown & 71.58 & 1.04 & $1.03,1.05$ & 29.92 & 0.97 & $0.95,0.99$ & 25.43 & 0.94 & $0.92,0.96$ & $13 \cdot 87$ & 0.99 & $0.95,1.02$ & 49.05 & 0.94 & $0.93,0.95$ \\
\hline Asian & $68 \cdot 38$ & 1.03 & $1.00,1.05$ & $29 \cdot 82$ & 0.97 & $0.93,1.02$ & $26 \cdot 88$ & 0.48 & $0.93,1.03$ & $14 \cdot 16$ & 1.03 & $0.96,1 \cdot 11$ & 48.42 & 0.95 & $0.93,0.98$ \\
\hline American Indian & $70 \cdot 51$ & 1.01 & $0.98,1.03$ & $30 \cdot 83$ & 1.03 & $0.98,1.08$ & 25.09 & 0.95 & $0.89,1.00$ & $14 \cdot 22$ & 1.02 & $0.94,1.11$ & $48 \cdot 15$ & 0.90 & $0.87,0.93$ \\
\hline \multicolumn{16}{|l|}{ Mother's educational level } \\
\hline Incomplete middle school & $72 \cdot 27$ & 1.00 & Ref. & 28.55 & 1.00 & Ref. & $23 \cdot 78$ & 1.00 & Ref. & $13 \cdot 22$ & 1.00 & Ref. & $46 \cdot 65$ & 1.00 & Ref. \\
\hline Complete middle school & 71.54 & 1.00 & $0.98,1.01$ & $31 \cdot 13$ & $1 \cdot 10$ & $1.07,1.13$ & $26 \cdot 2$ & $1 \cdot 11$ & $1.07,1 \cdot 14$ & $13 \cdot 33$ & 1.04 & $0.99,1.09$ & 51.58 & 1.06 & $1.04,1.08$ \\
\hline Complete high school & $69 \cdot 01$ & 1.00 & $9.98,1.00$ & $30 \cdot 50$ & $1 \cdot 10$ & $1.07,1.13$ & $27 \cdot 85$ & 0.05 & $1 \cdot 16,1 \cdot 22$ & $13 \cdot 13$ & 1.06 & $1.02,1.11$ & $55 \cdot 04$ & $1 \cdot 10$ & $1.08,1.12$ \\
\hline Complete higher education & $62 \cdot 70$ & 0.96 & $0.95,0.98$ & 33.34 & 1.25 & $1.21,1.29$ & 32.66 & 1.40 & $1.35,1.45$ & $15 \cdot 20$ & 1.28 & $1.21,1.34$ & $58 \cdot 16$ & 1.15 & $1 \cdot 13,1 \cdot 18$ \\
\hline \multicolumn{16}{|l|}{ Administrative status } \\
\hline Private & 58.87 & 1.00 & Ref. & 29.57 & 1.00 & Ref. & $29 \cdot 12$ & 1.00 & Ref. & $12 \cdot 87$ & 1.00 & Ref. & 56.90 & 1.00 & Ref. \\
\hline Public & $72 \cdot 23$ & $1 \cdot 19$ & $1 \cdot 18,1 \cdot 21$ & $30 \cdot 79$ & 1.08 & $1.05,1.11$ & $26 \cdot 05$ & 0.96 & $0.94,0.99$ & 13.58 & 1.07 & $1 \cdot 02,1 \cdot 11$ & $50 \cdot 44$ & 0.95 & $0.93,0.96$ \\
\hline \multicolumn{16}{|l|}{ Geographical area } \\
\hline North & 41.40 & 1.00 & Ref. & $26 \cdot 73$ & 1.00 & Ref. & $20 \cdot 99$ & 1.00 & Ref. & 13.90 & 1.00 & Ref. & 48.40 & 1.00 & Ref. \\
\hline Northeast & $70 \cdot 35$ & 1.42 & $1.39,1.44$ & 28.89 & 1.03 & $1.00,1.06$ & $24 \cdot 15$ & 0.95 & $0.92,0.98$ & 14.80 & 0.96 & $0.92,1.00$ & 39.90 & 0.86 & $0.85,0.88$ \\
\hline Southeast & 74.39 & 1.67 & $1 \cdot 65,1 \cdot 70$ & 31.68 & $1 \cdot 17$ & $1 \cdot 14,1 \cdot 21$ & $26 \cdot 12$ & $1 \cdot 19$ & $1 \cdot 15,1 \cdot 23$ & $13 \cdot 15$ & 0.96 & $0.92,1.01$ & 57.05 & 1.08 & $1.06,1 \cdot 10$ \\
\hline South & $69 \cdot 48$ & 1.32 & $1.30,1.35$ & 28.45 & 1.06 & $1.03,1 \cdot 10$ & 29.82 & 1.32 & $1.27,1.37$ & $10 \cdot 91$ & 0.82 & $0.78,0.87$ & $56 \cdot 61$ & $1 \cdot 11$ & $1.09,1.13$ \\
\hline Midwest & 73.30 & 1.62 & $1.59,1.65$ & 32.94 & $1 \cdot 19$ & $1 \cdot 15,1.22$ & 36.57 & 1.64 & $1.59,1.69$ & $15 \cdot 20$ & 1.03 & $0.98,1.08$ & 51.83 & 0.96 & $0.94,0.98$ \\
\hline \multicolumn{16}{|l|}{ County type } \\
\hline Capital & $60 \cdot 00$ & 1.00 & Ref. & $29 \cdot 76$ & 1.00 & Ref. & $27 \cdot 75$ & 1.00 & Ref. & $13 \cdot 85$ & 1.00 & Ref. & 54.64 & 1.00 & Ref. \\
\hline Non-capital & $72 \cdot 81$ & $1 \cdot 17$ & $1 \cdot 16,1 \cdot 19$ & $30 \cdot 34$ & 1.05 & $1.03,1.07$ & $26 \cdot 24$ & 0.98 & $0.96,1.00$ & $13 \cdot 35$ & 0.97 & $0.94,1.01$ & $50 \cdot 66$ & 0.91 & $0.90,0.92$ \\
\hline
\end{tabular}

PeNSE, Pesquisa Nacional de Saúde do Escolar (National Survey of Schoolchildren's Health); PR, prevalence ratio; Ref., referent category.

Adjusted for the other variables in the model. 
Table 3 Proportion of Brazilian adolescents who regularly consumed ( $\geq 5$ times/week) foods considered to be markers of an unhealthy diet according to sociodemographic variables; PeNSE 2012

\begin{tabular}{|c|c|c|c|c|c|c|c|c|c|c|c|c|c|c|c|c|c|c|}
\hline \multirow[b]{3}{*}{ Variable } & \multicolumn{18}{|c|}{ Unhealthy diet markers (consumed $\geq 5 \mathrm{~d} /$ week) } \\
\hline & \multicolumn{3}{|c|}{ Bagged salty snacks } & \multicolumn{3}{|c|}{ Fried salty snacks } & \multicolumn{3}{|c|}{ Processed meats } & \multicolumn{3}{|c|}{ Soft drinks } & \multicolumn{3}{|c|}{ Sweet biscuits } & \multicolumn{3}{|c|}{ Sweets } \\
\hline & $\%$ & $\mathrm{PR}^{*}$ & $95 \% \mathrm{Cl}$ & $\%$ & $\mathrm{PR}^{*}$ & $95 \% \mathrm{Cl}$ & $\%$ & $\mathrm{PR}^{*}$ & $95 \% \mathrm{Cl}$ & $\%$ & $\mathrm{PR}^{*}$ & $95 \% \mathrm{Cl}$ & $\%$ & $\mathrm{PR}^{*}$ & $95 \% \mathrm{Cl}$ & $\%$ & $\mathrm{PR}^{*}$ & $95 \% \mathrm{Cl}$ \\
\hline \multicolumn{19}{|l|}{ Gender } \\
\hline Male & $10 \cdot 93$ & 1.00 & Ref. & 14.17 & 1.00 & Ref. & $13 \cdot 72$ & 1.00 & Ref. & 33.59 & 1.00 & Ref. & $29 \cdot 72$ & 1.00 & Ref. & 33.79 & 1.00 & Ref. \\
\hline Female & 14.97 & 1.42 & $1.37,1.47$ & $17 \cdot 34$ & 1.24 & $1.21,1.28$ & $15 \cdot 51$ & $1 \cdot 16$ & $1 \cdot 11,1 \cdot 20$ & 32.89 & 1.01 & $0.99,1.03$ & 35.04 & $1 \cdot 20$ & $1 \cdot 18,1 \cdot 22$ & $48 \cdot 14$ & 1.41 & $1.39,1.43$ \\
\hline \multicolumn{19}{|l|}{ Age range } \\
\hline $11-14$ years & $12 \cdot 36$ & 1.00 & Ref. & $15 \cdot 22$ & 1.00 & Ref. & $14 \cdot 30$ & 1.00 & Ref. & $30 \cdot 86$ & 1.00 & Ref. & $32 \cdot 11$ & 1.00 & Ref. & 42.41 & 1.00 & Ref. \\
\hline 15 years or older & 14.51 & 1.26 & $1.21,1.30$ & $17 \cdot 11$ & 1.23 & $1 \cdot 19,1 \cdot 27$ & 14.85 & 1.13 & $1.09,1.77$ & 33.81 & $1 \cdot 18$ & $1 \cdot 16,1 \cdot 20$ & 33.34 & 1.05 & $1.03,1.07$ & 38.81 & 0.96 & $0.95,0.98$ \\
\hline \multicolumn{19}{|l|}{ Ethnicity/skin colour } \\
\hline White & $12 \cdot 02$ & 1.00 & Ref. & $15 \cdot 35$ & 1.00 & Ref. & $15 \cdot 20$ & 1.00 & Ref. & $34 \cdot 14$ & 1.00 & Ref. & 31.42 & 1.00 & Ref. & $40 \cdot 10$ & 1.00 & Ref. \\
\hline Black and brown & 13.62 & 1.06 & $1 \cdot 02,1 \cdot 10$ & 15.98 & 1.02 & $0.99,1.05$ & 14.38 & 0.99 & $0.96,1.03$ & 32.67 & 0.99 & $0.97,1.01$ & 33.25 & 1.04 & $1.02,1.06$ & $42 \cdot 16$ & 1.06 & $1.05,1.08$ \\
\hline Asian & $14 \cdot 16$ & 1.15 & $1.06,1.25$ & 17.53 & 1.11 & $1.04,1 \cdot 19$ & 13.99 & 1.10 & $1.02,1.18$ & 32.95 & 1.01 & $0.97,1.06$ & 31.62 & 1.05 & $1.01,1.10$ & 42.55 & 1.09 & $1.05,1.13$ \\
\hline American Indian & $13 \cdot 11$ & 1.08 & $0.99,1.18$ & $16 \cdot 27$ & 1.08 & $0.99,1.16$ & 14.02 & 1.04 & $0.96,1.13$ & 32.64 & 1.01 & $0.96,1.06$ & $32 \cdot 76$ & 1.05 & $1.00,1 \cdot 10$ & 38.19 & 1.02 & $0.97,1.06$ \\
\hline \multicolumn{19}{|l|}{ Mother's educational level } \\
\hline Incomplete middle school & 12.95 & 1.00 & Ref. & 14.64 & 1.00 & Ref. & $13 \cdot 22$ & 1.00 & Ref. & $30 \cdot 18$ & 1.00 & Ref. & 31.74 & 1.00 & Ref. & $40 \cdot 26$ & 1.00 & Ref. \\
\hline Complete $\mathrm{r}$ & 13.07 & 1.07 & $1.02,1 \cdot 13$ & $15 \cdot 87$ & 1.11 & $1.06,1 \cdot 16$ & 14.62 & 1.13 & $1.07,1 \cdot 18$ & 33.73 & 1.09 & $1.06,1 \cdot 13$ & $33 \cdot 14$ & 1.06 & $1.03,1.09$ & 41.28 & 1.03 & $1.01,1.06$ \\
\hline Complete $\mathrm{h}$ & $13 \cdot 19$ & 1.09 & $1.04,1.14$ & 16.57 & 1.18 & $1 \cdot 13,1.22$ & $15 \cdot 67$ & 1.21 & $1 \cdot 16,1 \cdot 26$ & $35 \cdot 30$ & $1 \cdot 14$ & $1 \cdot 11,1 \cdot 17$ & 33.42 & 1.09 & $1.06,1.12$ & 42.05 & 1.06 & $1.04,1.08$ \\
\hline Complete higher education & 12.89 & 1.16 & $1.09,1.23$ & 17.57 & 1.23 & $1.17,1.30$ & 16.69 & 1.23 & $1 \cdot 16,1 \cdot 29$ & $36 \cdot 86$ & $1 \cdot 13$ & $1.09,1.16$ & 31.68 & 1.09 & $1.05,1.12$ & $42 \cdot 55$ & 1.04 & $1.02,1.07$ \\
\hline \multicolumn{19}{|l|}{ Administrative status } \\
\hline Private & 11.39 & 1.00 & Ref. & 17.46 & 1.00 & Ref. & $15 \cdot 83$ & 1.00 & Ref. & $37 \cdot 28$ & 1.00 & Ref. & $30 \cdot 32$ & 1.00 & Ref. & 40.98 & 1.00 & Ref. \\
\hline Public & $13 \cdot 38$ & $1 \cdot 18$ & $1 \cdot 12,1 \cdot 24$ & 15.48 & 0.87 & $0.84,0.90$ & 14.41 & 0.89 & $0.85,0.92$ & $32 \cdot 38$ & 0.93 & $0.91,0.95$ & 32.95 & 1.08 & $1.06,1.11$ & 41.34 & 1.03 & $1.01,1.05$ \\
\hline \multicolumn{19}{|l|}{ Geographical areas } \\
\hline North & 9.96 & 1.00 & Ref. & $15 \cdot 37$ & 1.00 & Ref. & 9.39 & 1.00 & Ref. & 28.87 & 1.00 & Ref. & $26 \cdot 02$ & 1.00 & Ref. & 36.60 & 1.00 & Ref. \\
\hline Northeast & 12.85 & 1.24 & $1 \cdot 18,1.31$ & $16 \cdot 34$ & 1.04 & $1.00,1.08$ & 12.53 & 1.38 & $1.32,1.45$ & $28 \cdot 20$ & 0.89 & $0.87,0.92$ & 31.43 & 1.29 & $1 \cdot 26,1 \cdot 33$ & 39.49 & 1.03 & $1.00,1.05$ \\
\hline Southeast & 14.60 & 1.28 & $1 \cdot 22,1 \cdot 36$ & 16.47 & 0.96 & $0.92,1.01$ & $17 \cdot 04$ & 1.60 & $1.51,1.68$ & $38 \cdot 27$ & $1 \cdot 16$ & $1 \cdot 13,1 \cdot 19$ & $35 \cdot 22$ & 1.36 & $1.32,1.40$ & $42 \cdot 30$ & $1 \cdot 13$ & $1 \cdot 10,1 \cdot 16$ \\
\hline South & $10 \cdot 74$ & 1.04 & $0.97,1.11$ & $14 \cdot 13$ & 0.89 & $0.85,0.94$ & 14.93 & 1.54 & $1.46,1.63$ & $28 \cdot 18$ & 0.97 & $0.94,1.00$ & $30 \cdot 00$ & 1.13 & $1.09,1.17$ & 41.73 & $1 \cdot 11$ & $1.08,1.14$ \\
\hline Mid-west & $12 \cdot 25$ & $1 \cdot 18$ & $1.12,1.24$ & 14.08 & 0.91 & $0.87,0.95$ & $12 \cdot 87$ & 1.26 & $1.20,1.33$ & 34.65 & 1.14 & $1 \cdot 11,1 \cdot 17$ & 31.78 & 1.24 & $1.20,1.27$ & $45 \cdot 14$ & $1 \cdot 19$ & $1.16,1.22$ \\
\hline \multicolumn{19}{|l|}{ County type } \\
\hline Capital & 11.99 & 1.00 & & 15.74 & 1.00 & Ref. & 14.90 & 1.00 & Ref. & 35.44 & 1.00 & Ref. & 31.92 & 1.00 & Ref. & $42 \cdot 62$ & 1.00 & Ref. \\
\hline Non-capital & $13 \cdot 34$ & $1 \cdot 12$ & $1.08,1 \cdot 16$ & $15 \cdot 84$ & 1.03 & $1.00,1.06$ & 14.59 & 0.91 & $0.88,0.93$ & 32.58 & 0.92 & $0.90,0.94$ & 32.67 & 1.03 & $1.01,1.05$ & 40.89 & 0.97 & $0.96,0.99$ \\
\hline
\end{tabular}

PeNSE, Pesquisa Nacional de Saúde do Escolar (National Survey of Schoolchildren's Health); PR, prevalence ratio; Ref., referent category.

${ }^{*}$ Adjusted for the other variables in the model. 
Table 4 Association of the nutritional scale score with sociodemographic variables and health-risk and health-protective behaviours in Brazilian adolescents attending the ninth year of basic education; PeNSE 2012

\begin{tabular}{|c|c|c|c|c|}
\hline \multirow[b]{2}{*}{ Variable } & \multicolumn{4}{|c|}{ Nutritional scale score* } \\
\hline & Coefficient $(\beta)$ & SE & $P$ value & $95 \% \mathrm{Cl}$ \\
\hline Gender $($ male $=0 ;$ female $=1)$ & $-1 \cdot 60$ & $0 \cdot 11$ & 0.000 & $-1.83,-1.38$ \\
\hline \multicolumn{5}{|l|}{ Age range $(11-14$ years $=0)$} \\
\hline 15 years or older & -0.06 & 0.09 & 0.467 & $-0.24,-0.11$ \\
\hline \multicolumn{5}{|l|}{ Ethnicity/skin colour (white $=0$ ) } \\
\hline Black and brown & -0.08 & 0.20 & 0.695 & $-0.47,0.32$ \\
\hline Asian & -0.03 & 0.17 & 0.856 & $-0.36,0.30$ \\
\hline American Indian & 0.33 & 0.46 & 0.474 & $-0.58,1.25$ \\
\hline \multicolumn{5}{|l|}{ Mother's educational level (incomplete middle $=0$ ) } \\
\hline Complete middle school & -0.33 & $0 \cdot 10$ & 0.001 & $-0.52,-0.13$ \\
\hline Complete high school & -0.46 & 0.14 & 0.001 & $-0.74,-0.18$ \\
\hline Complete higher education & -0.33 & 0.19 & 0.080 & $-0.70,0.04$ \\
\hline \multicolumn{5}{|l|}{ Meals with parents (never/seldom $=0$ ) } \\
\hline $1-2 d$ & 0.75 & 0.33 & 0.024 & $0.10,1.41$ \\
\hline $3-4 \mathrm{~d}$ & 0.75 & 0.25 & 0.003 & $0.25,1.25$ \\
\hline $5 \mathrm{~d}$ or more & 1.91 & 0.28 & 0.000 & $1 \cdot 36,2 \cdot 45$ \\
\hline \multicolumn{5}{|l|}{ Eating while studying or watching TV (never/seldom $=0$ ) } \\
\hline $1-2 d$ & $-1 \cdot 71$ & 0.27 & 0.000 & $-2 \cdot 24,-1 \cdot 18$ \\
\hline $3-4 d$ & $-2 \cdot 77$ & 0.22 & 0.000 & $-3 \cdot 19,-2 \cdot 34$ \\
\hline $5 \mathrm{~d}$ or more & -4.45 & 0.13 & 0.000 & $-4 \cdot 70,-4 \cdot 20$ \\
\hline \multicolumn{5}{|l|}{ Eating breakfast (never/seldom $=0$ ) } \\
\hline $1-2 d$ & $1 \cdot 14$ & 0.21 & 0.000 & $0.73,1.55$ \\
\hline $3-4 d$ & 1.03 & 0.34 & 0.003 & $0.35,1.70$ \\
\hline $5 \mathrm{~d}$ or more & 2.84 & $0 \cdot 15$ & 0.000 & $2.55,3.13$ \\
\hline Smoked in the past $30 \mathrm{~d}($ no $=0$; yes $=1$ ) & -1.43 & 0.14 & 0.000 & $-1 \cdot 70,-1 \cdot 15$ \\
\hline Alcohol in the past $30 \mathrm{~d}(\text { no }=0 \text {; yes }=1)^{\prime}$ & -2.99 & 0.08 & 0.000 & $-3.15,-2.82$ \\
\hline Drugs in the past $30 \mathrm{~d}($ no $=0$; yes $=1)$ & -2.09 & 0.20 & 0.000 & $-2 \cdot 47,-1.71$ \\
\hline Physically active $\dagger$ (inactive $=0$; active $=1$ ) & 1.27 & 0.07 & 0.000 & $1 \cdot 13,1.40$ \\
\hline School administrative status (private $=0$; public $=1$ ) & 1.06 & 0.21 & 0.000 & $0.65,1.47$ \\
\hline \multicolumn{5}{|l|}{ Geographical areas (North $=0$ ) } \\
\hline Northeast & 0.50 & 0.89 & 0.574 & $-1 \cdot 24,2 \cdot 24$ \\
\hline Southeast & 0.92 & 0.79 & 0.243 & $-0.62,2.47$ \\
\hline South & 1.69 & 0.90 & 0.061 & $-0.08,3.46$ \\
\hline Mid-west & $2 \cdot 71$ & 1.00 & 0.007 & $0.75,4.66$ \\
\hline County type (capital $=0$; non-capital $=1$ ) & 0.29 & 0.22 & 0.196 & $-0.15,0.72$ \\
\hline
\end{tabular}

PeNSE, Pesquisa Nacional de Saúde do Escolar (National Survey of Schoolchildren's Health); TV, television.

*The total score was calculated by adding the scores from the items measuring the frequency of intake of healthy foods - fruits, raw and cooked vegetables, milk and beans $(0=$ never to $7=$ every day) - and subtracting the frequency of intake of unhealthy foods - sweets, sweet biscuits, bagged salty snacks, fired salty snacks and cold meats $(0=$ every day to $7=$ never).

†The participants who spent at least $300 \mathrm{~min}$ on gym classes at school, sports, leisure physical activities and active commuting in the previous week were considered active.

adolescents who are exposed to various health hazards and the assessment of dietary intake can help to identify these groups.

A study of adolescents from five Asian countries found that an absence of family-related protective factors (lack of family ties and parental supervision) and physical inactivity were associated with lower fruit and vegetable intake $^{(19)}$. Cluster analysis and investigation of behavioural patterns have been performed in adults ${ }^{(20,21)}$. Future studies to identify risky and protective behaviours among Brazilian adolescents might be interesting. In addition, such studies might help establish which and how sociodemographic characteristics are associated with risky and protective behavioural patterns, leading to interventions targeting the groups at highest risk.

In our study, the girls exhibited a similar frequency of intake of healthy foods and a higher frequency of intake of unhealthy foods in comparison to boys; therefore the girls presented, on average, 1.60 less points on the nutritional scale. Although the frequency of regular intake of unhealthy foods was slightly higher among the older participants, the nutritional scale score was not correlated with age. These findings suggest that early interventions promoting healthy nutrition should take into account the greater exposure of girls to undesirable dietary habits, as well as the need to increase the frequency of regular intake of healthy foods in both genders.

Our results agree with the results from the Brazilian state capitals in PeNSE $2009^{(12)}$ as well as with the results in adolescents attending public schools in the city of Rio de Janeiro ${ }^{(15)}$. Comparisons with the international literature should be made cautiously because of the indicators used. Nevertheless, it is possible to compare the correlations and differences involving gender, age and economic level between the present study and other studies.

Undesirable dietary profiles and poorer dietary intake with increased age have also been found in Europe ${ }^{(22)}$ and the USA $^{(23)}$. Data from the US Youth Risk Behaviour 
Surveillance System (YRBSS) for 2011 show that $64.0 \%$ and $62.3 \%$ of youths consumed fruits/natural fruit juice and vegetables, respectively, at least once daily. In contrast to our study, those data indicate greater intake of those foods by boys $(66 \cdot 1 \%$ and $62 \cdot 8 \%$ of boys regularly consuming fruits and vegetables, respectively). Daily intake of soft drinks was found in $27 \cdot 8 \%$ of youths in that study, also most frequently in boys $(31.4 \%)^{(23)}$. Data from the European Health Behaviour in School-aged Children (HBSC) survey of adolescents aged 11, 13 and 15 years in thirty-nine countries indicate that inadequate dietary patterns occurred more frequently among the older and the male adolescents. The proportion of daily intake of fruit was lower among the 15-year-olds (31\%) compared with the 11-year-old group ( $42 \%$ ). The proportion of daily intake of soft drinks was higher among the oldest adolescents ( $25 \%$ in 15-year-olds $v .18 \%$ in 11-year-olds). A multicentre study conducted with European adolescents found that the average intake of fruits and vegetables was about half that recommended in the guidelines of the Optimized Mixed Diet and Food Guide Pyramid, and this dietary inadequacy was more frequent among the boys. In addition, excessive intakes of oil, fat and sweets were also found in both genders ${ }^{(24)}$.

The positive association of the intake of both healthy and unhealthy foods with the mother's educational level found in the present study may possibly be explained by the development of responsibility for health-related behaviours and attitudes among teenagers. Individuals' autonomy about their food choice increases during adolescence and parents occupy a less important role, in addition to competing with the external influences of the adolescents' peers ${ }^{(25,26)}$. Although research among children has found a protective effect of maternal education on obesity ${ }^{(27)}$, studies among adolescents found results similar to ours ${ }^{(12,23)}$.

The public schools had a greater proportion of individuals who consumed beans and fruits on a regular basis. In addition, the score on the nutritional scale was higher among the public school students compared with those attending private schools $(\beta=1 \cdot 06)$. These results might be a consequence of the school food plans established by the National Program of Schoolchildren Nutrition. That programme is enforced by law and supervised by nutritionists, and one of its goals is to promote healthy nutritional habits ${ }^{(28)}$. The Program for Health at School is a more recent initiative devoted to the promotion of health among schoolchildren, targeting public schools only ${ }^{(29)}$. However, the proportion of regular intake of bagged salty snacks and sweet biscuits was higher among the public school students compared with the students in private schools. As the public school students eat only a part of their daily meals at school, public policies should include strategies targeting families and the adolescents' family environment to increase their effectiveness $^{(30)}$.
The results of the present study should be assessed only after taking some possible limitations into consideration. The data on the nutritional scale should be interpreted cautiously because in the elaboration of that scale, the same weight was attributed to the intake of healthy and unhealthy foods, whereas low intake of healthy foods might not have the same biological effects as high intake of unhealthy foods. In addition, small correlation values were found even among unhealthy or healthy items, and this scenario reinforces the need for using several indicators of healthy and unhealthy food consumption in adolescents. Nevertheless, the internal consistency of the scale attained the minimum acceptable value and the direction of its association with sociodemographic and behavioural variables agrees with reports in the literature. It is also worth noting that since the size of the PeNSE sample guaranteed a high statistical power some associations reached statistical significance even when comparing slightly different prevalences between groups, thus the borderline associations shown in Tables 3 and 4 must be read taking this into consideration.

With regard to the external validity of PeNSE, it should be remembered that it used a representative sample of Brazilian children and adolescents enrolled in 9th grade from public and private schools. When the wide coverage of basic education in Brazil is taken into account - 97\% and $88 \%$ of the population aged 6-14 and $15-19$ years old, respectively - it is safe to assume that the total adolescent population of Brazil exhibits the same patterns of behaviour $^{(31)}$. In addition, the rates of no response to PeNSE were low. Moreover the sample coverage was $83 \%$, which is considered adequate, and the factors of expansion used in the analyses were recalculated by taking the coverage losses into consideration.

The results of the present study might serve as a basis for public-health measures and broaden the scope of the strategies that have been implemented to promote health among adolescents in the Brazilian school setting. In this regard, the present system of surveillance and monitoring of the health-risk and health-protective factors in adolescents should be maintained and reinforced to provide an accurate picture of the tendencies and magnitudes of these factors, to (re)orient local actions and public policies.

\section{Acknowledgements}

Financial support: The present research received financial support from USP/FM/PROAP CAPES/PROAP - 055/2013 Medicina Preventiva/CGC.63·025.530/0018-52 for manuscript translation and formatting. C.M.A. received a doctoral scholarship from Conselho Nacional de Desenvolvimento Científico e Tecnológico (CNPq). L.F.M.d.R. received a masters scholarship from Coordenação de Aperfeiçoamento de Pessoal de Nivel Superior (CAPES). The funders had no role in study design, data collection and analysis, decision to 
publish, or preparation of the manuscript. Conflict of interest: None. Authorship: C.M.A., R.B.L. and D.S.C conceptualized the study and were involved in the analysis and interpretation of the results. L.F.M.d.R was involved in data preparation and designed and prepared the tables. The initial draft of the paper was prepared by C.M.A. following extensive discussions with I.R.R.d.C., R.B.L., R.M.C. and O.d.C.L. Successive drafts were developed by C.M.A., D.S.C. and L.F.M.d.R., with inputs from the other co-authors. All authors have reviewed and approved the final version. Ethics of buman subject participation: PeNSE 2012 was approved by the National Commission of Research Ethics (Comissão Nacional de Ética em Pesquisa (CONEP); record no. 16 805) and was performed in accordance with the Declaration of Helsinki. All participants gave their informed consent. Its database was made publicly available at the Brazilian Institute of Geography and Statistics' website without any information allowing the identification of participants.

\section{References}

1. Hosseinpoor AR, Bergen N, Kunst A et al. (2012) Socioeconomic inequalities in risk factors for non communicable diseases in low-income and middle-income countries: results from the World Health Survey. BMC Public Health 12, 912.

2. Schmidt MI, Duncan BB, Azevedo e Silva G et al. (2011) Chronic non-communicable diseases in Brazil: burden and current challenges. Lancet 377, 1949-1961.

3. Martins APB, Levy RB, Claro RM et al. (2013) Increased contribution of ultra-processed food products in the Brazilian diet (1987-2009). Rev Saude Publica 47, 1-10.

4. Monteiro CA, Levy RB, Claro RM et al. (2011) Increasing consumption of ultra-processed foods and likely impact on human health: evidence from Brazil. Public Health Nutr 14, 5-13.

5. World Health Organization (2003) Diet, Nutrition and the Prevention of Chronic Diseases. Report of a Joint WHO/FAO Expert Consultation. WHO Technical Report Series no. 916. Geneva: WHO.

6. Levy RB, Claro RM, Mondini L et al. (2012) Regional and socioeconomic distribution of household food availability in Brazil, in 2008-2009. Rev Saude Publica 46, $6-15$.

7. Moubarac JC, Martins AP, Claro RM et al. (2013) Consumption of ultra-processed foods and likely impact on human health. Evidence from Canada. Public Health Nutr 16, 2240-2248.

8. Baş M, Altan T, Dinçer D et al. (2005) Determination of dietary habits as a risk factor of cardiovascular heart disease in Turkish adolescents. Eur J Nutr 44, 174-182.

9. Adrian M, Charlesworth-Attie S, Vander Stoep A et al. (2014) Health promotion behaviors in adolescents: prevalence and association with mental health status in a statewide sample. J Behav Health Serv Res 41, 140-152.

10. Birkhead GS, Riser MH, Mesler K et al. (2006) Youth development is a public health approach. Introduction. J Public Health Manag Pract Suppl., S1-S3.

11. Ministério do Planejamento, Orçamento e Gestão, Instituto Brasileiro de Geografia e Estatística \& Diretoria de Pesquisas, Coordenação de População e Indicadores Sociais (2013) Pesquisa Nacional de Saúde do Escolar 2012 (National Survey of Schoolchildren's Health 2012). Rio de Janeiro: IBGE.
12. Levy RB, de Castro IRR, Cardoso LdO et al. (2010) Consumo e comportamento alimentar entre adolescentes brasileiros: Pesquisa Nacional de Saúde do Escolar (PeNSE), 2009 (Food intake and dietary behaviour among Brazilian adolescents: National Survey of Schoolchildren's Health (PeNSE), 2009). Cien Saude Colet 15, 3085-3097.

13. Tavares LF, de Castro IRR, Levy RB et al. (2014) Validade relativa de indicadores de práticas alimentares da Pesquisa Nacional de Saúde do Escolar entre adolescentes do Rio de Janeiro, Brasil. Cad Saude Publica 30, 1029-1041.

14. World Health Organization (2004) Global Strategy on Diet, Physical Activity and Health: Fifty-Seventh World Health Assembly. Geneva: WHO.

15. de Castro IRR, Cardoso LO, Engstrom EM et al. (2008) Vigilância de fatores de risco para doenças não transmissíveis entre adolescentes: a experiência da cidade do Rio de Janeiro, Brasil (Surveillance of risk factors for non-communicable diseases in adolescents: experience in the city of Rio de Janeiro, Brazil). Cad Saude Publica 24, 2279-2288.

16. World Health Organization (2010) Global Recommendations on Physical Activity for Health. Geneva: WHO.

17. Royston P \& White IR (2011) Multiple imputation by chained equations (MICE): implementation in Stata. J Stat Softw 45, 1-20.

18. Barros AJ \& Hirakata VN (2003) Alternatives for logistic regression in cross-sectional studies: an empirical comparison of models that directly estimate the prevalence ratio. BMC Med Res Methodol 3, 21.

19. Peltzer K \& Pengpid S (2012) Fruits and vegetables consumption and associated factors among in-school adolescents in five Southeast Asian countries. Int $J$ Environ Res Public Health 9, 3575-3587.

20. Galán I, Rodríguez-Artalejo F, Tobías A et al. (2005) Clustering of behavior-related risk factors and its association with subjective health. Gac Sanit 19, 370-378.

21. Steele EM, Claro RM \& Monteiro CA (2014) Behavioural patterns of protective and risk factors for noncommunicable diseases in Brazil. Public Health Nutr 17, 369-375.

22. Currie C, Roberts C, Morgan A et al. (editors) (2012) Social Determinants of Health and Well-Being Among Young People. Health Behaviour in School-aged Children (HBSC) Study: International Report from the 2009/2010 Survey. Copenhagen: WHO Regional Office for Europe.

23. Eaton DK, Kann L, Kinchen S et al.; Centers for Disease Control and Prevention (2011) Youth risk behavior surveillance - United States, 2011. MMWR Surveill Summ 61, 1-162.

24. Diethelm K, Jankovic N, Moerno LA et al. on behalf of the HELENA Study Group (2012) Food intake of European adolescents in the light of different food-based dietary guidelines: results of the HELENA (Healthy Lifestyle in Europe by Nutrition in Adolescence) Study. Public Health Nutr 15, 386-398.

25. Brown K, Mcllveen H \& Strugnell C (2006) Nutritional awareness and food preferences of young consumers. Nutr Food Sci 30, 230-235.

26. Contento IR, Williams SS, Michela JL et al. (2006) Understanding the food choice process of adolescents in the context of family and friends. J Adolesc Health 38, $575-582$.

27. Lamerz A, Kuepper-Nybelen J, Wehle C et al. (2005) Social class, parental education, and obesity prevalence in a study of six-year-old children in Germany. Int J Obes (Lond) 29, 373-380.

28. Ministério da Educação, Fundo Nacional de Desenvolvimento da Educação, Coordenação Geral do Programa Nacional de Alimentação Escolar (2012) Manual de Orientação para a Alimentação Escolar na Educação Infantil, Ensino Fundamental, Ensino Médio e na 
Educação de Jovens e Adultos (Manual of Orientation for School Nutrition in Pre-school Education, Elementary School, Secondary School and Youth and Adult Education), 2nd ed. Brasília: CECANE-SC.

29. Presidência da República Casa Civil Subchefia para Assuntos Jurídicos (2007) Decreto $n^{\circ}$. 6.286, de 5 de dezembro de 2007 [Decree no. 6.286 from December 5 2007]. http://www.planalto.gov.br/ccivil_03/_Ato2007-2010/ 2007/Decreto/D6286.htm (accessed November 2013).
30. Cullen KW, Watson K \& Zakeri I (2008) Improvements in middle school student dietary intake after implementation of the Texas Public School Nutrition Policy. Am J Public Health 98, 111-117.

31. Instituto Brasileiro de Geografia e Estatística (n.d.) Pesquisa Nacional por Amostra de Domicílio (PNAD) 2008/ 2011. v. 29/31, 2008-2012. http://www.ibge.gov.br/home/ estatistica/populacao/trabalhoerendimento/pnad2011/default. shtm (accessed November 2013). 